\title{
Positron annihilation and constant photocurrent method measurements on a-Si:H films: A comparative approach to defect identification
}

\author{
P.M. Gordo ${ }^{\mathrm{a}, *}$, M.F. Ferreira Marques ${ }^{\mathrm{b}}$, C. Lopes Gil ${ }^{\mathrm{a}}$, A.P. de Lima ${ }^{\mathrm{a}}$, \\ G. Lavareda ${ }^{c}$, C. Nunes de Carvalho ${ }^{\text {c }}$, A. Amaral ${ }^{\text {c }}$, Zs. Kajcsos $^{\mathrm{d}}$ \\ ${ }^{a}$ Departamento de Física, Universidade de Coimbra, Portugal

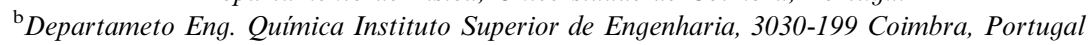 \\ ${ }^{\mathrm{c}}$ Centro de Fisica Molecular, IST/UTL, Lisboa, Portugal \\ ${ }^{\mathrm{d}}$ KFKI Research Institute for Nuclear and Particle Physics, Budapest, Hungary
}

\begin{abstract}
Defect structure of hydrogenated amorphous silicon thin-films was studied by positron annihilation spectroscopy (PAS), whereas the density of states below the Fermi level was measured by constant photocurrent method (CPM). Divacancies and large vacancy clusters were identified as the main defects present in these films, with relative concentrations strongly dependent on the rf-power. Correlation between PAS, CPM results and $I(V)$ characteristics of solar cells suggests the creation of energy levels above the Fermi energy, not observable by CPM, related to large vacancy clusters.
\end{abstract}

(C) 2006 Elsevier Ltd. All rights reserved.

Keywords: Variable-energy positron annihilation; Doppler broadening; Constant photocurrent method; Defects; a-Si:H; Density of states

\section{Introduction}

Hydrogenated amorphous silicon (a-Si:H) is a thinfilm semiconductor with increasing importance from both technological and scientific points of view. It has a wide variety of applications in microelectronics and optoelectronics, particularly in thin film transistors for photovoltaics and display applications. The most commonly used deposition technique for industrial production of a-Si:H films is plasma enhanced chemical vapor deposition (PECVD) (Abelson, 1993).

\footnotetext{
*Corresponding author. Fax: +351239829158 .

E-mail address: pgordo@ci.uc.pt (P.M. Gordo).
}

The electrical and optical properties of the a-Si:H films are controlled by the localized states in the band gap, the dangling bond being the principal defect state inside the band gap of a-Si:H. Dangling bonds are most likely associated with small multivacancy complexes (divacancies, trivacancies, etc.) within the disordered tetrahedrally coordinated network.

In this work, the density of a-Si:H electronic deep defects was studied by CPM measurements; the techniques of positron annihilation spectroscopy (PAS) were used to investigate the defect structure, particularly vacancy type defects. In order to better understand the correlation between both results, the performance of solar cells based on a-Si:H thin-films was also studied by $I(V)$ measurements. 
PAS features techniques suited for the study of the electron density and electron momentum density around the defects. These methods might be employed in the study of semiconductors (Krause-Rehberg and Leipner, 1999a) as bulk techniques or in low-energy positron experiments for near-surface and thin-film studies like in our case, performing Doppler broadening experiments using a slow positron beam (Lima et al., 1999). In defect studies, the annihilation lineshape is often characterized in terms of two parameters, $S$ and $W$ e.g. (KrauseRehberg and Leipner, 1999b), corresponding to the fractions of annihilation with low-momentum (valence) and high-momentum (core) electrons, respectively. The low-momentum fraction, $S$, depends primarily on size and concentration of open volume defects, whereas the high-momentum fraction, $W$, is sensitive to the electronic structure at the annihilation site. Together, these two parameters form a unique signature for different defect structures (Liszkay et al., 1994).

The constant photocurrent method (CPM) (Vanecek et al., 1981), was used to measure the subgap absorption coefficient of the amorphous semiconductor samples. In $\mathrm{CPM}$, the impinging monochromatic photon flux is adjusted, at each wavelength, so as to maintain a constant photocurrent. In the assumption of a constant conduction electron generation rate the inverse of the photon flux is proportional to the absorption coefficient, $\alpha_{\text {CPM }}$. The density of deep states (DOS) can be determined from the sub-band-gap absorption spectra in a-Si:H using the "single energy" technique (Wyrsch et al., 1991).

\section{Experimental}

The a-Si:H films were deposited by magnetically confined $\mathrm{rf}(13.56 \mathrm{MHz})$ PECVD on crystalline silicon (c-Si) for PAS studies, and on alkali-free glass substrates for CPM measurements, according to the following optimised deposition conditions: substrate temperature, $T_{\mathrm{S}}=250^{\circ} \mathrm{C}$; deposition pressure, $200 \mathrm{mTorr} ; \mathrm{SiH}_{4}$ flow, $F_{\mathrm{SiH}_{4}}=20 \mathrm{sccm}$ and magnetic field, $B=160 \mathrm{G}$. The rf power, $P$, varied between 5 and $40 \mathrm{~W}$. The thickness of the films was about $600 \mathrm{~nm}$ for all the samples. The positron beam Doppler broadening measurements were performed varying the positron energies from 0.1 to $30 \mathrm{keV}$ (Lima et al., 1999) on the a-Si:H films deposited on c-Si. The DOS values were determined from CPM spectra (Wyrsch et al., 1991) using a Kracos monochromator with light intensity adjustment. The density of deep defects was calculated from $N_{\mathrm{dd}}=C_{\text {ref }} \times \alpha\left(E_{\text {ref }}\right)$, with $C_{\text {ref }}=10^{16} \mathrm{~cm}^{-2}$ and $E_{\text {ref }}=1.2 \mathrm{eV}$.

Solar cells based on intrinsic a-Si:H with the structure (ITO $/ \mathrm{p}-i-\mathrm{n} / \mathrm{Al}$ ) were also prepared. Indium tin oxide (ITO) films were deposited by reactive thermal evapora- tion (RTE). Aluminium films were deposited by thermal evaporation. Undoped a-Si:H films were deposited using rf powers of 10,20 and $30 \mathrm{~W}$ under the previously referred conditions. Doped $\mathrm{n}$ and $\mathrm{p}$ layers were deposited also by PECVD using $\mathrm{PH}_{3}$ or $\mathrm{B}_{2} \mathrm{H}_{6}$ and $\mathrm{CH}_{4}$ as dopants, respectively.

\section{Results and discussion}

The Doppler parameters, $S_{\text {film }}$ and $W_{\text {film }}$, and the positron diffusion length, $L_{+}$, of each a-Si:H film deposited on c-Si were obtained fitting the $S(\mathrm{E})$ and $W(\mathrm{E})$ data using VEPFIT (van Veen et al., 1990) assuming samples consisting of two layers, one being the a-Si:H film and the c-Si substrate the other.

Fig. 1a shows the $S_{\text {film }}$ parameter normalized to $S_{\mathrm{c}-\mathrm{Si}}$ for various a-Si:H films deposited at different rf powers. The normalized $S_{\text {film }}$ parameter reaches a maximum already at a relatively low rf power value and decreases then steadily. On the other hand, the linear behaviour of the $S_{\text {film }}-W_{\text {film }}$ plot (Fig. 1b) strongly suggests that in these films two types of open volume defects are present, leading to competing trapping at a near-saturation level. The relative concentration of those trapping sites is dependent of the rf power deposition. The experimental boundary values of the $S_{\text {film }}$ are $\sim 1.027$ and $\sim 1.06$. In
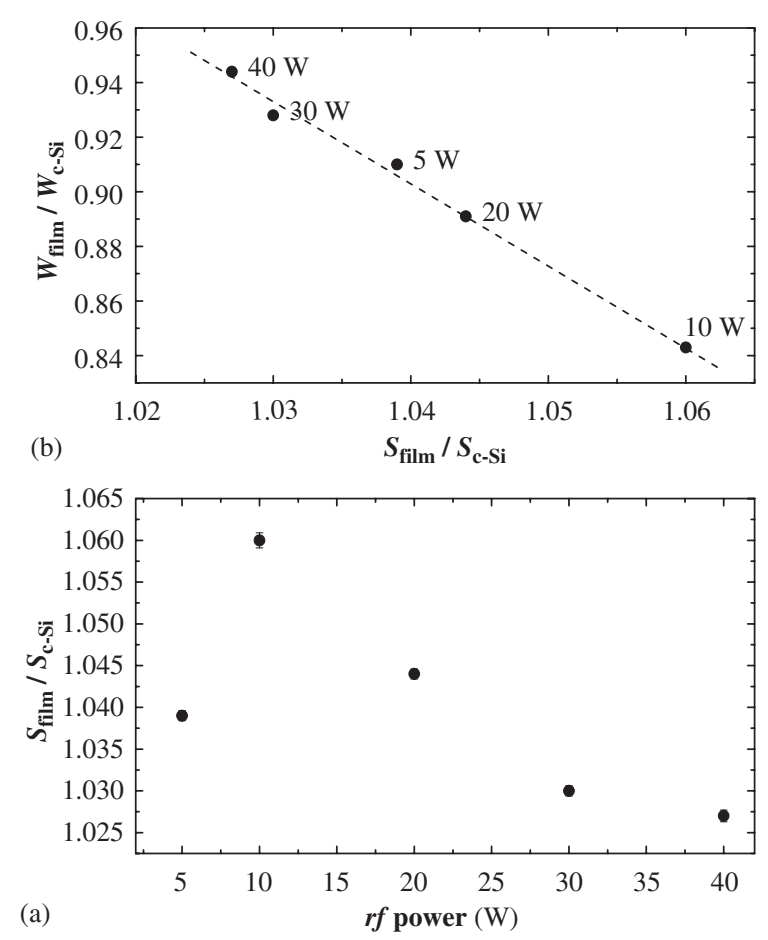

Fig. 1. (a) Normalized $S_{\text {film }}$ and (b) $S-W$ plot, for films deposited at different $\mathrm{rf}$ powers. 

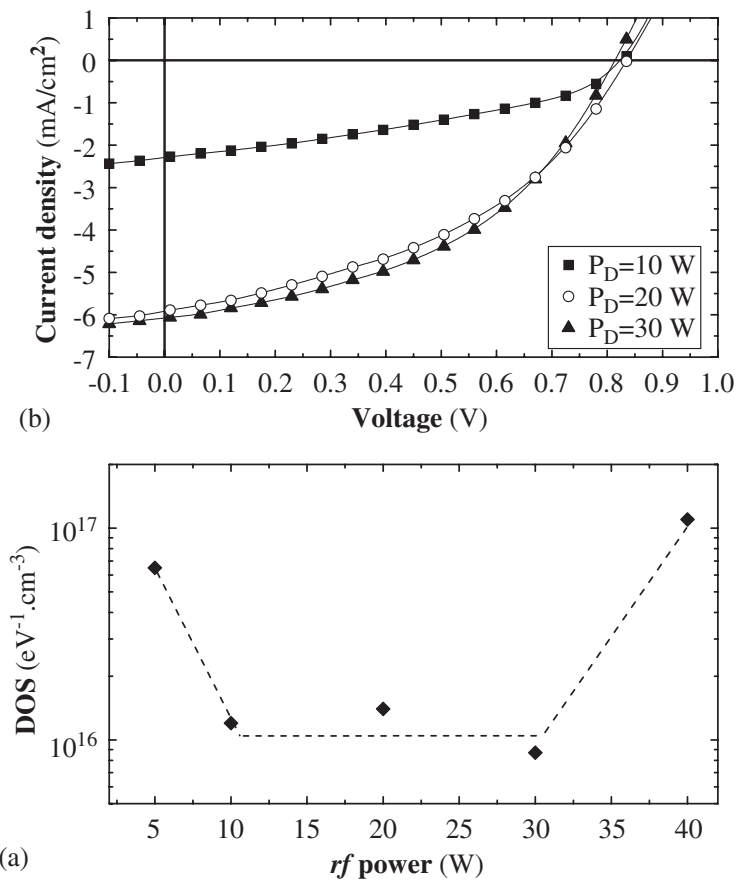

Fig. 2. (a) Deep states density as a function of the rf deposition power. (b) Current density vs. applied voltage for the solar cells prepared with a-Si:H films.

principle, four defect types which may trap positrons can be considered in a-Si:H (Zou et al., 2000; Amarendra, 2002): monovacancy, divacancy, small vacancy cluster (with $\sim 5 \mathrm{Si}$ atoms missing) and large vacancy clusters or microvoids with a diameter of 1-2 nm, where positronium (Ps) can also be formed. Indeed, our maximum and minimum $S_{\text {fim }}$ values might correspond to trapping in large vacancy clusters/microvoids and in small vacancy type defects (probably divacancies), respectively. The highest $S_{\text {film }}$ value may also include contributions from positron surface states and from Ps caged inside cavities.

Fig. 2a presents the variation with the rf power of DOS (below $E_{\mathrm{F}}$ ) as obtained from CPM measurements. For the lowest and the highest rf powers the DOS values are about one order of magnitude higher than in the range of $10-30 \mathrm{~W}$, where the films reach the typical value for good quality films $\left(10^{16} \mathrm{eV}^{-1} \mathrm{~cm}^{-3}\right)$. The highest DOS values observed at 5 and $40 \mathrm{~W}$ may be interpreted by an incomplete passivation of the dangling bonds either due to insufficient hydrogen incorporation (as expected at the lowest $\mathrm{rf}$ power) or due to the strong damage caused by the ion bombardment leading to the bond-breaking or to the release of hydrogen previously incorporated (as expected to occur at high rf power). The films produced at powers between 10 and $30 \mathrm{~W}$ exhibit quite different defect structures as observed by PAS, whereas the DOS values indicate similar deep state densities below the $E_{\mathrm{F}}$. The film deposited at $10 \mathrm{~W}$ reveals large vacancy clusters as the main positron traps while at $30 \mathrm{~W}$ divacancies are dominant.

The influence of these defect types on the optoelectronic properties of a-Si:H films was also studied. The current density as a function of the applied voltage under AM1 illumination conditions was measured on solar cells prepared with a-Si:H films grown at rf powers of 10,20 and $30 \mathrm{~W}$ maintaining the other deposition conditions constant. In Fig. $2 \mathrm{~b}$ the $V-I$ curves obtained for these solar cells are presented. The poorest cell performance is observed on the solar cell prepared with $10 \mathrm{~W}$, and the best performance is achieved for the film fabricated with $30 \mathrm{~W}$. The high concentration of large vacancy-type defects in the $10 \mathrm{~W}$ film results in a lower current density. Two reasons can contribute to this behaviour: the film with higher concentration of large vacancy clusters has a lower mass density and consequently the incident light produces less electron-hole pairs per unit volume and an increase of the DOS above the Fermi level. This fact has indeed been reported before (Babras et al., 1990) in a-Si:H films studied by deep level transient spectroscopy, photoluminescence and spectroscopic ellipsometry. These levels, related to large vacancy clusters, may also act as charge recombination centres and are not attainable by CPM, which determines the DOS below the Fermi level. Moreover, the incident $1.2 \mathrm{eV}$ photons not only promote electron transitions from deep states inside the bandgap to the conduction band (being the dominant contribution to the measured photocurrent), but can also promote electronic transitions from the valence band up to those levels related to large vacancy clusters, which do not contribute to the photocurrent measured by CPM (Schmidt and Rubinelli, 1998; Schmidt et al., 2000). Consequently, for higher concentrations of large vacancy clusters a higher light intensity is needed to keep the generated photocurrent constant but leading then to an underestimation of the DOS value when determined by this method.

\section{Conclusions}

PAS was used to characterize the defect structure of aSi:H films deposited by PECVD on c-Si substrates. Two dominant defect types were identified, large vacancy clusters and divacancies. The DOS below $E_{\mathrm{F}}$ was measured by CPM using the "single energy technique". From the comparison of PAS and CPM results with the performance of solar cells, the existence of states above the Fermi energy and inside the bandgap, related to the presence of large vacancy clusters in a-Si:H films, was evidenced. These levels influence the recombination of charge carriers, cause an underestimation of DOS values determined by "single energy" CPM measurements, and 
contribute to the weaker performance of a-Si:H solar cells with high concentration of large vacancy clusters.

\section{Acknowledgements}

The support provided by the Hungarian-Portuguese Bilateral Scientific agreement TET No. P23/03 is gratefully acknowledged.

\section{References}

Abelson, J.R., 1993. Plasma deposition of hydrogenated amorphous silicon: studies of the growth surface. Appl. Phys. A 56, 493-512.

Amarendra, G., 2002. Positron annihilation studies of vacancy defects in crystalline and amorphous Si. Defects and Diffusion in Semiconductors IV 200-202, 189-217.

Babras, S., Rajarshi, S.V., Dusane, R.O., Bhide, V.G., Kshirsagar, S.T., 1990. Effect of rf power on the structure and related gap states in hydrogenated amorphous silicon. J. Non-Cryst. Solids 119, 342-346.

Krause-Rehberg, R., Leipner, H.S., 1999a. Positron Annihilation in Semiconductors-Defect Studies. Springer, Berlin, Heidelberg, pp. 1-4.

Krause-Rehberg, R., Leipner, H.S., 1999b. Positron Annihilation in Semiconductors-Defect Studies. Springer, Berlin, Heidelberg, pp. 21-24.

Lima, A.P. de, Lopes Gil, C., Gordo, P.M., Duarte Naia, M., 1999. Initial results with a variable energy positron system at Coimbra. In: OECD/NEA (Ed.), Workshop on Ion and
Slow Positron Beam Utilization, Costa da Caparica, Portugal, pp. 121-128.

Liszkay, L., Cordel, C., Baroux, L., Hautojärvi, P., Bayhan, M., Brinkmann, A.W., Tatarenko, S., 1994. Positron trapping at divacancies in thin polycrystalline CdTe films deposited on glass. Appl. Phys. Lett. 64, 1380-1382.

Schmidt, J.A., Rubinelli, F.A., 1998. Limitations of the constant photocurrent method: a comprehensive experimental and modelling study. J. Appl. Phys. 83, 339-348.

Schmidt, J.A., Koropecki, R.R., Arce, R.D., Rubinelli, F.A., Buitrago, R.H., 2000. Energy-resolved photon flux dependence of the steady state photoconductivity in hydrogenated amorphous silicon: implications for the constant photocurrent method. Thin Solid Films 376, 267-274.

Vanecek, M., Kocka, J., Stuchlík, J., Triska, A., 1981. Direct measurement of the gap states and tail absorption by constant photocurrent method in amorphous silicon. Solid State Commun. 39, 1199-1202.

van Veen, A., Schut, H., Hakvoort, J., Vries, R.A., Ijpma, M.R., 1990. Analysis of positron Profiling Data by Means of "VEPFIT". In: Schultz, P.J., Massoumi, G.R., Simpson, P.J. (Eds.), Positron Beams for Solids and Surfaces. AIP Conference Proceedings, vol. 218. London, Ontario, Canada, pp. 171-196.

Wyrsch, N., Finger, F., McMahon, T.J., Vanecek, V., 1991. How to reach more precise interpretation of subgap absorption spectra in terms of deep defect density in aSi:H. J. Non-Cryst. Solids 137-138, 347-350.

Zou, X., Chan, Y.C., Webb, D.P., Lam, Y.W., Hu, Y.F., Beling, C.B., Fung, S., Weng, H.M., 2000. Photoinduced dehydrogenation of defects in undoped a-Si:H using positron annihilation spectroscopy. Phys. Rev. Lett. 84, 769-772. 